\title{
UNA FUENTE INÉDITA DE LA DOCTRINA DE LA SUPOSICIÓN EN VICENTE FERRER: LA POLÉMICA BURLEIGH \& OCKHAM
}

\author{
José Ángel García Cuadrado \\ Universidad de Navarra
}

\section{RESUMEN}

La polémica entre Guillermo de Ockham y Walter Burleigh en el siglo XIV propició la aparición de tratados independientes sobre la doctrina de la suposición, como el Textus de Suppositionibus de William Sutton. Este tratado presenta coincidencias muy significativas con el Tractatus de Suppositionibus de Vicente Ferrer; estas coincidencias llevan a formular la hipótesis de una influencia directa de Sutton en Ferrer.

Palabras clave: Ockham, Burleigh, Sutton, Vicente Ferrer, suposición.

\begin{abstract}
The $14^{\text {th }}$ century controversy between William of Ockham and Walter Burleigh led to the publication of autonomous treatises on the theory of supposition, such as William Sutton's Textus de Suppositionibus. Significant parallels may be found between this treatise and the Tractatus de Suppositionibus of Vincent Ferrer; such coincidences lead to the formulation of the hypothesis of a direct influence of Sutton on Ferrer.
\end{abstract}

Key words: Ockham, Burleigh, Sutton, Vincent Ferrer, Supposition.

A lo largo de los últimos años han aparecido diversos trabajos de investigación en los que se recogía la original doctrina de la suposición contenida en los tratados lógicos de San Vicente Ferrer. ${ }^{1}$ La novedad de los planteamientos ferrerianos parece obedecer a una personal elaboración del pensamiento tomista, como el mismo dominico valenciano reconoce de modo explícito en diversos pasajes de sus obras. No obstante, un detenido análisis textual nos lleva a pensar que no sólo se sirvió de la doctrina de Tomás de Aquino, sino que conocía también los tratados lógicos de

1 Cfr. López García, A., «Nota para la historia de la lingüística española: la contribución de San Vicente Ferrer» en Philologica II (1989), pp. 389-398; Beuchot, M., La filosofia del lenguaje en la Edad Media, México, UNAM, 1984; Id. El pensamiento filosófico de San Vicente Ferrer; Valencia, Ayuntamiento de Valencia, 1995; García Cuadrado, J. A., Hacia una semántica realista. La filosofia del lenguaje de San Vicente Ferrer, Pamplona, Eunsa, 1994. Puede consultarse también la bibliografía que se encuentra en id. «Los tratados filosóficos de $S$. Vicente Ferrer: nota histórica y bibliográfica», en Revista Española de Filosofia Medieval, 1 (1994), pp. 61-73. 
su tiempo. En este trabajo, analizaré de modo pormenorizado las fuentes textuales de las que se sirvió nuestro autor para elaborar sus tratados lógicos. El fruto de nuestra investigación nos revelará además algunos aspectos del ambiente filosófico de nuestra Península en el otoño del periodo medieval.

De esta época apenas tenemos alguna noticia reveladora. En ocasiones, de muchos autores y de sus obras apenas nos ha llegado alguna referencia a través de autores posteriores. Esta falta de datos históricos sobre nuestro pensamiento, que afecta particularmente a los siglos XIII y XIV, resulta comprensible si tenemos en cuenta que una vez pasado el esplendor cultural del siglo XII, el núcleo del pensamiento filosófico se desplaza a otros centros culturales, concretamente a las universidades de Oxford y París.

La Península Ibérica vive en gran parte al margen del florecimiento filosófico medieval. ${ }^{2} \mathrm{La}$ dependencia de la Universidad parisina es enorme. Los autores de este período pertenecen casi siempre a alguna de las distintas órdenes religiosas, de entre los cuales, los más sobresalientes marchan a París para completar allí sus estudios. Algunos de ellos permanecerán allí como maestros de Teología. Los que regresan a la Península ejercen una influencia en un ámbito reducido y sus obras - teológicas o filosóficas- alcanzan escasa difusión. Por otro lado, el contenido doctrinal parece ser poco original acusando excesivamente la dependencia de los grandes maestros (Alberto Magno, Tomás de Aquino, Buenaventura, Duns Escoto, etc...). Esta visión general apenas esbozada y necesariamente muy simplificada, explicaría porqué conocemos tan poco de los filósofos hispanos medievales, relegados al olvido de los manuales, permaneciendo sus obras inéditas o desconocidas.

Afortunadamente, la vida y obra del maestro Ferrer nos resultan bastante conocidas pues se trató de una figura destacada en la vida política y religiosa de su tiempo. No obstante, hasta comienzos del siglo XX sus tratados lógicos nos eran completamente desconocidos. Desde entonces, y de manera creciente han despertado el interés y la admiración de los historiadores de la lógica por su notable originalidad, alejada de las doctrinas lógicas más aceptadas en su tiempo.

\section{LOS TRATADOS LÓGICOS DEL MAESTRO FERRER}

Vicente Ferrer fue el autor de dos tratados filosóficos: la Questio de Unitate Universalis y el Tractatus de Suppositionibus. Se trata de dos obras de juventud que pertenecen a su período de formación dentro de la Orden de Predicadores. Si acudimos a los datos históricos podremos encontrar algunos indicios acerca de las circunstancias que motivaron estos tratados. Ferrer nació en Valencia en el año $1350 .{ }^{3}$ En 1367 tomó el hábito en el convento dominicano de esa ciudad y en febrero de

2 «La valiosísima aportación que significó la labor de los traductores del siglo XII, y que tuvo tan profundas repercusiones en la orientación y desarrollo de la escolástica en París y Oxford, apenas revela su huella en la España del siglo XIII [....]. No hallamos nada que pueda parangonarse con lo que, a base de los mismos elementos suministrados por las traducciones toledanas, realizará por ese tiempo la escolástica en Francia y otros países, ni figuras que de lejos se puedan comparar con un San Alberto, un San Buenaventura ni un Santo Tomás». Fraile, G., Historia de la Filosofía española (I). Desde la época romana hasta fines del siglo XVII, (2ª edición), Madrid, BAC, 1985, p. 153.

3 Cfr. Garganta, J.M., y Forcada, V., Biografia y escritos de San Vicente Ferrer, Madrid, BAC, 1956; Gorce, M. M., voz «Vincent Ferrier», en Dictionnaire de Theologie Catholique, París, 1950, t. XV, cols. 3033-3045. 
1368 profesa los votos solemnes y comienza su formación en Artes. Ese mismo año, en septiembre, se traslada a Barcelona como estudiante de Lógica. Una vez terminado el curso 1368/69, se le asigna como alumno de los Studium naturarum en el convento de Lérida. Durante los cursos 1370/71 y 1371/72 es nombrado lector de Lógica (magister logicorum) en la Universidad de Lérida. En el curso 1372 se traslada a Barcelona para completar sus estudios bíblicos y teológicos. En el curso $1374 / 75$ es profesor de naturales, trasladándose en 1376 a Toulouse para terminar su formación teológica. Alcanzó unos años después el rango de Maestro en Teología, la máxima distinción académica de entonces.

Por los datos que nos han llegado a través de los manuscritos, los tratados filosóficos pertenecen a la etapa de su enseñanza de Lógica en Lérida, es decir cuando nuestro autor contaba sólo con veintidós años y era un simple encargado de dictar las lecciones a los jóvenes novicios. ${ }^{4}$ Read apunta acertadamente que nuestro autor debería tratarse de un joven y hábil profesor, que reelaboró el material tradicional para sus estudiantes del convento de Lérida. ${ }^{5}$ Sin embargo, como él mismo reconoce, conocemos muy poco de esta tradición lógica que se había fraguado durante este periodo. Si es cierto que el Tractatus de Suppositionibus del dominico valenciano, no es más que la reelaboración de doctrinas lógicas innovadoras, sería sin duda alguna interesante reconstruir las fuentes de esa nueva tradición.

Por lo que respecta al Estudio General de Lérida hay que decir que se trató de la primera universidad de la Corona de Aragón, fundada en el año 1300. A pesar de ser un enclave geográfico excelente para los reinos de Castilla, Navarra, Aragón y Cataluña, no llegó a alcanzar rango europeo; sólo en el siglo XV se le concede la facultad de Teología. Su esplendor se corresponde con la primera mitad del siglo XIV; después decayó por divisiones internas y por la creación de nuevas universidades cercanas: Perpiñán (1350) y Huesca (1354). Desde el siglo XIV existía en la universidad ilerdense una cátedra libre de Teología encomendada a los franciscanos.

Sin embargo, más que la universidad de Lérida, son las Escuelas conventuales de las diversas órdenes religiosas las que mejor podrían trasmitir las nuevas ideas filosóficas y teológicas. ${ }^{6}$ En tiempos de Vicente Ferrer, en Lérida encontramos una Escuela conventual de dominicos desde 1230 - donde impartiría sus lecciones nuestro autor-, y otra de franciscanos. Este dato es significativo, porque mientras que la Orden de Predicadores había ya adoptado en 1309 las obras de Tomás de Aquino como texto de Teología, Ios Franciscanos se encontraban divididos entre los escotistas y ockhamistas, sin olvidar a los lulistas, con gran fuerza en toda la Corona de Aragón. ${ }^{\text {? }}$

4 Una exposición del estado de la cuestión acerca de la cronología de estas obras se encuentra en García Miralles, M., «Escritos filosóficos de San Vicente Ferrer», en Estudios Filosóficos, 4 (1955), pp. 279-284; Poveda, E., «El tratado De Suppositionibus dialecticis de S. Vicente Ferrer y su significación histórica en la cuestión de los universales», en Anales del Seminario de Valencia, 6 (1963), pp. 5-88.

5 «Ferrer's treatise is that of a young teacher, a young and very clever teacher, reworking traditional material for his students at the convent in Lerida». Read, S.L., «Thomas of Cleves and Collective Supposition», en Vivarium, 29 (1991), p. 74.

6 Cfr. Carreras Artau, J. y T., Historia de la Filosofia Española (II). Filosofia cristiana de los siglos XIII al XV, Madrid, CSIC, 1943, pp. 60-65 y pp. 76-77.

7 «Quiere esto decir que las corrientes doctrinales, en tiempos de Vicente Ferrer, llevaban el signo del tomismo, por una parte, y el del escotismo-ockhamismo-lulismo, por otra [...] La lucha entre lulianos y tomistas está candente en Lérida, como también está viva la lucha entre tomistas y escotistas, lo mismo que las corrientes nominalistas». Forcada, V., «Momento histórico del tratado De Suppositione de San Vicente Ferrer», en Escritos del Vedat, 3 (1973), pp. 45-46. 
De los maestros de San Vicente tenemos escasas noticias. De su corta preparación en Valencia poco sabemos, excepto que era obligatoria una primera formación en la gramática y retórica, antes de ingresar en el convento. En el curso 1368/69, durante su estancia en Barcelona, tuvo como lector de Lógica a Esteban Miquel, ${ }^{8}$ pero no tenemos ningún dato más sobre este autor. En 1369 , en Lérida recibió las enseñanzas en Filosofía de Bernardo Descoll, del que sabemos también pocas cosas. Los biógrafos del santo hablan además de Bernardo Armengol ( $†$ 1387) como uno de los maestros de Ferrer durante su estancia barcelonesa. ${ }^{9}$ También nos hablan de un tal Juan Linconiense, del convento de Lérida «que escribió muy bien sobre los cuatro libros del Maestro de las Sentencias, y sobre la Lógica y la Filosofía recopiló otros opúsculos pequeños». ${ }^{10}$ No tenemos más datos biográficos de él salvo que pudo ser contemporáneo de Ferrer o inmediatamente anterior. Por último, podríamos citar a Antonio de Ginebreda (+1395), compañero de San Vicente en Barcelona (1368/69) y posteriormente profesor de Filosofía en Lérida en 1371. ${ }^{11}$

De estos autores prácticamente desconocidos no parece a primera vista que Ferrer recibiera un influjo directo y decisivo. Pero de lo que sí podemos estar seguros es de su formación aristotélicotomista, y que al menos dos de ellos conocían perfectamente los comentarios de Santo Tomás a los libros de la Sentencias, en donde encontramos de manera explícita aplicaciones de la teoría de la suposición en vistas a resolver los problemas cristológicos. En este contexto marcadamente teológico es donde se desenvolverá la doctrina de la suppositio, tanto en Tomás de Aquino como en el maestro Ferrer. ${ }^{12}$

Una última anotación biográfica puede servirnos para comprender mejor la propuesta ferreriana. El Maestro Ferrer no estudió en París, como hicieron los maestros dominicos más conocidos de su tiempo. De esta manera, sus estudios de Lógica no estuvieron mediatizados por el Tractatus de Pedro Hispano. En efecto, las Summulae Logicales fueron escritas entre 1230 y 1245 ; posteriormente fueron adoptadas como texto en la Universidad parisina y se mantuvo en las universidades europeas hasta bien entrado el siglo XV. Por lo tanto, la tradición escrita de Ferrer no bebió, al menos directamente, de la tradición terminista parisina. ¿Cual fue entonces la tradición lógica que recibió el dominico valenciano? Quizás, el estudio de las fuentes citadas en sus tratados pueda arrojarnos algo de luz sobre este asunto.

8 Cfr. Fages, H., Notes et documents de l'Histoire de St. Vincent Ferrer, Louvain, 1905, pp. 38-39.

9 «(Armengol) escribió doctísimos comentarios sobre los cuatro libros del Maestro de las Sentencias». Diago, F., Historia de la Provincia de Aragón de la Orden de Predicadores, desde su origen y principio hasta el año mil y seiscientos [...], Barcelona 1599, fol. 56. Citado por Forcada, V., «Momento histórico [...]» p. 48. Cfr. también, Díaz Díaz, G., Hombres y documentos de la Filosofia española (I), Madrid, CSIC, 1980, p. 375.

10 Diago, F., Historia de la Provincia [...], fol. 151; Robles, L., Escritores dominicos de la Corona de Aragón: siglosXIIIXV, Salamanca, 1972, p. 279; Díaz Díaz, G., Hombres y documentos de la Filosofía española (IV), Madrid, CSIC, 1991, p. 705 .

11 Cfr. Robles, L., Escritores dominicos [...] , pp. 146-148.

12 Cfr. Brown, S., «Medieval Supposition Theory in its Theological Context», en Medieval Philosophy and Theology 3 (1993), 121-157; Maierù, A., «A propos de la doctrine de la supposition en théologie trinitarie au XIV siècle», en Medieval Semantics and Metaphysics, Nijmegen, Ed. E.P. Bos, Ingenium Publishers, 1985, pp. 221-238. 


\section{FUENTES TEXTUALES DE LOS TRATADOS LÓGICOS DEL MAESTRO FERRER}

Vicente Ferrer es bastante explícito a la hora de citar las fuentes antiguas y medievales en las que se basa su argumentación, recurriendo sistemáticamente a las autoridades reconocidas para avalorar sus afirmaciones. Sin embargo, apenas gusta de citar a autores contemporáneos suyos, aunque sin duda debió recibir de ellos alguna influencia. El análisis de las fuentes citadas expresamente por nuestros autor puede servirnos para reconstruir parcialmente el marco intelectual donde se encuadran los tratados vicentinos.

En cuanto a las fuentes citadas en la Quaestio De Unitate Universalis, hemos de distinguir las fuentes explícitas y las implícitas. El autor más citado es Aristóteles (en 24 ocasiones cita expresamente sus obras). Sólo citará en una ocasión a Boecio, Avicena y el Liber de Causis. El profesor Trentman ha hecho notar la ausencia de referencias explícitas a Santo Tomás, pero hemos de reconocer que la doctrina ferreriana es deudora por completo de la del Doctor Angélico. ${ }^{13}$ No podemos dudar de la filiación aristotélico-tomista de Ferrer a la hora de abordar la cuestión de los universales, que será imprescindible para comprender la doctrina de la suposición de los términos.

El análisis de las fuentes mencionadas en el tratado De Suppositionibus viene a confirmar la filiación aristotélico-tomista del dominico valenciano. Aparte de otras citas no explícitas, encontramos las siguientes: Aristóteles (74 citas); Santo Tomás (39); Averroes (5). Otros autores tan sólo se citan en una ocasión: Boecio, Porfirio, al gramático Pedro Elías, ${ }^{14}$ Alfarabí, Egidio Romano así como el Liber de Causis. A estas citas hay que añadir la mención a Walter Burleigh y Guillermo de Ockham de la que posteriormente hablaré.

Por otro lado, las referencias a la suposición impropia revelan un cierto conocimiento de la Retórica que quizás aprendiera en su primera formación. ${ }^{15}$ Pero, con todo, no parece que se trate de una influencia demasiado decisiva. También resulta interesante comprobar las citas de los filósofos árabes. Hemos de tener en cuenta que en la Corona de Aragón los intercambios culturales y filosóficos entre cristianos y musulmanes fueron muy fructíferos, registrándose mutuas influencias. ${ }^{16}$ Pero, por otro lado, es muy probable que las obras de los filósofos musulmanes llegaran en gran parte a través de las obras y comentarios aristotélicos compuestos por Santo Tomás.

13 Cfr. Trentman, J.A., «The Questio De Unitate Universalis of Vincent Ferrer», en Medieval Studies, 44 (1982), p. 115 .

14 La cita del gramático Pedro Elías, es interesante pues basándose en su definición de Gramática sienta los fundamentos para la distinción entre el gramático y el lógico: mientras que el primero estudia las palabras y las voces, el lógico tiene por objeto los contenidos mentales. Cfr. Trentman, J. A., "Vincent Ferrer on the Logician as Artifex Intellectualis», en Frasciscan Studies, 25 (1965), pp. 322-337.

15 López García ha hecho notar la coincidencia entre la propuesta ferreriana y las doctrinas de los modistas. Como él mismo reconoce, se tratan de coincidencias debidas a un fondo doctrinal común, pero no obedece a una influencia directa. Cfr. López García, A., «Nota para la historia...», pp. 389-398.

16 Cfr. Lomba Fuentes, J., «El papel de la Frontera Superior en la Trasmisión del pensamiento islámico a Europa», en Diálogo filosófico-religioso entre cristianismo, judaísmo e Islamismo durante la Edad Media en la Península Ibérica, Actes du colloque intemational de San Lorenzo de Escorial. 23-26 juin 1991, Brèpols 1994, Turnhout, p. 396. Cfr. también Lomba Fuentes, J., «La trasmisión del saber andalusí a Europa en la Corona de Aragón», en Mediaevalia. Textos e Estudos, 7-8 (1995), pp. 199-220. 
Junto a los autores citados, resulta también muy revelador lo que calla el Maestro Ferrer. AI tratar de la suposición de los términos, no cita directamente ningún tratado lógico de su tiempo: ni el Tractatus de Pedro Hispano, ni las Introductiones in Logicam de Shyreswood, ni la Summula de Lambert de Auxerre, por tan sólo citar algunos de los tratados más difundidos entre los escolares de su época. Sólo en el prólogo a su tratado sobre las suposiciones dará los nombres (no las obras) de dos lógicos anteriores a su magisterio: Burleigh y Ockham. Pienso que no resultará infructuoso preguntarnos por estos autores, los únicos contemporáneos citados por Vicente Ferrer.

\section{EL DEBATE BURLEIGH \& OCKHAM}

Las citas de estos dos autores está situada en las primeras líneas del Tractatus, donde Ferrer manifiesta su propósito con estas palabras: «[...] porque Walter Burleigh trató de las suposiciones según la primera opinión extrema del universal; y, porque trató también según la otra opinión extrema el hermano Guillermo de Ockham y sus seguidores, mucho más se habrá de decir del universal y tratar de las suposiciones, según la sentencia verdadera y media (de Santo Tomás)».17

En este texto, quiero subrayar en primer lugar la relación que Ferrer establece de modo explícito entre teoría de la suposición y el problema de los universales. En efecto, la doctrina de la suposición de los términos no es un problema meramente conceptual o de análisis lógico, sino que en esta doctrina se dilucidan las cuestiones acerca del alcance gnoseológico y ontológico de los conceptos universales.

Más significativa todavía es la mención explícita de Vicente Ferrer a las posturas de Ockham y Burleigh. Está bastante documentada la polémica que ambos autores mantuvieron en las primeras décadas del siglo XIV, tanto en la cuestión de los universales como en la teoría de la suposición. ${ }^{18}$

Walter Burleigh (1275-1349?), Doctor planus et perspicuus, enseñó en Oxford, París y Toulouse. ${ }^{19}$ Mantenía la existencia real del universal en una postura similar a la defendida por Duns Escoto: ${ }^{20}$ por esta razón, aparece en los manuales de Historia de la Filosofía como un escotista peculiar. ${ }^{21}$ De acuerdo con estos principios escotistas elaboró un primer tratado De Suppositionibus en 1301 como fruto de su enseñanza en Oxford en la Facultad de Artes. La Summa Logica de

17 Utilizaré la traducción de Forcada, citando por la página de esa edición, pero añadiendo entre paréntesis las páginas correspondientes a la edición de Trentman, Tractatus de Suppositionibus, Grammatica Speculativà, Sprachtheorie und Logik des Mittelalters 2: Stuttgart-Bad Cannstatt 1977. TS, p. 45 (88).

18 Mccord Adams, M., «Universals in the Early Fourteenth Century», en The Cambridge History of Later Medieval Philosophy, Cambridge University Press 1982, pp. 411-439; Spade, P.V., «Some epistemological implications of the Burley \& Ockham Dispute», en Franciscan Studies, 35 (1975), pp. 212-222; Wagner, P., «Supposition-Theory and the problem of universals (W. Burleigh \& W. Ockham)», en Franciscan Studies, 41 (1981), pp. 385-414. Un reciente y documentado estado de la cuestión acerca de las relaciones entre Ockham y Burleigh se encuentra en Larre, O., «Walter Burley: las primeras reacciones contra Ockham desde el realismo», en Estudios Franciscanos, 98 (1997), pp. 11-28.

19 Cfr, Uña Juaréz, A., La filosofia del siglo XIV. Contexto cultural de Walter Burley; Madrid, Real Monasterio del Escorial, Biblioteca «La Ciudad de Dios», 1978.

20 Crr. De Libera, A., Philosphie médiévale, Paris, P.U.F., 1995, pp. 441-442.

21 Cfr. Fraile, G., Historia de la Filosofia, (II-2), Madrid, BAC, 1986, p. 530; De Wulf, M., Historia de la Filosofia Medieval, (III), México, JUS, 1949, pp. 148-150; Gilson, E., La Filosofia en la Edad Media, Madrid, Gredos, 1976, p. 572. 
Guillermo de Ockham aparece en 1323, en donde realiza una abierta crítica de la noción de suppositio de Burleigh, especialmente en lo que se refiere a la suppositio simplex, aunque se muestra también un influjo positivo en algunas cuestiones, como la inclusión de los relativos dentro de la suposición, así como el tratamiento de la suposición material. ${ }^{22}$

Las críticas de Ockham hicieron que Walter Burleigh revisara su obra de juventud y hacia 1329 apareció el De Puritate Artis Logicae, en la que profundiza más en su propuesta marcando sus diferencias con el nominalismo ockhamiano, manteniendo sin embargo intacto gran parte de su anterior tratado: las coincidencias textuales entre el primer y el segundo tratado resultan significativas.

Pues bien, pienso que Vicente Ferrer no era ajeno a esta polémica cuando elaboró sus tratados filosóficos, unos cuarenta años después. Poveda, al tratar de la Questio de Unitate Universalis hace notar que se encuentra especialmente dirigida contra los realistas exagerados y que los argumentos contra esta postura ofrecen bastantes paralelismos con el comentario al I Libro de las Sentencias llevado a cabo por Guillermo de Ockham. Aunque se tratara de argumentos comúnmente empleados en las discusiones lógicas, no habría que descartar la lectura directa del texto ockhamiano a la hora de redactar Ferrer esta cuestión, sin olvidar, claro está, que su postura es la vía media, la del realismo de Tomás de Aquino.

Sin embargo, el Tractatus de Suppositionibus parece especialmente dirigido hacia los nominales. Los tratados de Walter Burleigh muestran asombrosas semejanzas con el del Maestro Ferrer, precisamente en algunos de los puntos más originales de su doctrina. En primer lugar, resulta extraña la coincidencia de títulos entre el primer tratado de Burleigh y la obra del dominico valenciano. No es una cuestión sólo de nombres, sino también de novedad metodológica, pues hasta el tratado de Burleigh la teoría de la suposición de los términos se había estudiado siempre dentro de las propiedades de los términos y éstas junto a otros tratados sobre De consequentiis, De obligatoriis o insolubilibus, etc. Está documentado que la aparición de los tratados sobre las suposiciones de modo independiente es un fenómeno característico de la Facultad de Artes de Oxford. En efecto, hacia 1330-1340 William Sutton, lector del Menton College, compuso un Textus De Suppositionibus, en el que nos informa que la exposición de manera independiente de las suposiciones, se debe al prolijo tratamiento que de esta materia realizaron Burleigh y Ockham, de tal manera que se vio conveniente confeccionar un tratado para principiantes en las escuelas lógicas de Oxford. ${ }^{23}$

22 Cfr. Brown, S., «Walter Burleigh's Treatise De Suppositionibus and his influence on William of Ockham», en Frasciscan Studies, 32 (1972), pp. 15-64.

23 «Because of the prolixity of both Ochkam and Burley (nimis esse prolixa) some simple treatise had to be established for beginner in the Oxford Schools of logic». Weisheipl, J.A., «Developments in the Arts Curriculum at Oxford in the Early Fourteenth Century», en Mediaeval Studies, 28 (1966), p. 158. En la nt. 28 de este artículo se recoge este interesante testimonio de Sutton: «Sed causa efficiens (tractatus supposicionum) fuit Wilhelmus Okham et Walterus Werley, ambo anglici, qui sentencia prolixa tradiderunt scienciam de supposicionibus terminorum, et hoc quantum ad causam principalem. Sed alteri modernorum videntur dicta sua nimis esse prolixa et viris anglicis qui colligit illum librum de supposicionibus ex libris aliorum de quo nomine nichil curretur». Scriptum magistri Sifridi anglici super textum de supposicionibus Sutonis Anglici. Vienna, Dominikanerkloster MS 160/130, fol. 91vb-92ra. 
Otras muchas semejanzas pueden detectarse en un análisis comparativo más detenido. Por ejemplo, al tratar de la noción de suppositio, Burleigh en el De Puritate Artis Logicae, comienza distinguiendo tres sentidos: «La suposición, como generalmente se entiende es la acepción del término por algo, ya sea por una cosa, por una voz o por un concepto». ${ }^{24}$ Literalmente esta es la definición que Ferrer rechaza y es - según él- la peor de todas las definiciones que se han dado. «Entre todas las definiciones o descripciones que se dan comúnmente de la suposición, la menos suficiente es aquella que dice: la suposición es la acepción o uso del término categórico que se toma por algo o por varias cosas en la proposición». ${ }^{25}$ ¿Qué otras definiciones tenía nuestro autor a la vista? Creo que las que está aduciendo Burleigh.

La segunda definición — continúa Burleigh — dice así: «La suposición, considerada comúnmente, es la propiedad del término comparado con el otro término en la proposición. Y de este modo la suppositio compete tanto al sujeto como al predicado, [...] y es más amplia que la apelación, porque la suposición compete tanto al sujeto como al predicado mientras que la apelación compete sólo al predicado». ${ }^{26}$ Esta definición no la hemos encontrado en ningún tratado anterior, si exceptuamos el Tractatus de Suppositionibus de Burleigh. Ferrer no la recoge en su texto, pero afirma explícitamente que el predicado sólo posee apelación. ${ }^{27}$

Pero Burleigh añade una tercera definición más propia que la anterior: «La suposición, en sentido propio, es la propiedad del término sujeto comparado al predicado». ${ }^{28}$ Aquí tenemos, casi de modo literal, la misma definición que Ferrer nos presenta con estas palabras: «La suposición es la propiedad del sujeto comparado con el predicado en la proposición». ${ }^{29}$ De lo anterior se deduce que sólo el sujeto supone, y no el predicado, como ya afirmara Burleigh. Y en este punto, Burleigh y Ferrer se separan de toda la tradición parisina, desde Pedro Hispano, Guillermo de Ockham y Alberto de Sajonia. «Siendo así que la suposición es una propiedad del sujeto y sólo a él compete, es manifiesto que el predicado no tendrá suposición alguna». ${ }^{30}$ Ferrer no presenta dudas en su afirmación y añade: «ni puede decirse que, aunque el predicado no suponga con suposición propiamente dicha, sin embargo supone con suposición tomada en sentido amplio». ${ }^{31}$ Es precisamente lo que Walter Burleigh estaba dispuesto a conceder cuando admite en sentido amplio, la primera definición de suppositio.

24 «Suppositio generaliter dicta est acceptio termini pro aliquo, scilicet pro re vel pro voce vel pro conceptu». Walter Burleigh, De Puritate Artis Logicae Tractatus Longior, ed. Ph. Boehner, New York, The Franciscan Institute St. Bonaventure, 1955, (en adelante DPAL) p. 2. La definición se corresponde con la de Pedro Hispano, pero todavía más iiteralmente con la propuesta por Lambert de Auxerrre.

25 TS, p. 55 (94). Posteriormente matizará su afirmación diciendo que esta definición no es completamente errónea pero no le parece suficiente por diversos motivos que explica con detalle.

26 «Suppositio communiter accepta est proprietas termini ad alium terminum in propositione comparati. Et isto modo convenit suppositio tam subiecto quam praedicato quam etiam verbo seu consignificatis ipsius verbi. Et suppositio hoc modo accepta est in plus quam appellatio, quia suppositio competit tam subiecto quam praedicato et appellatio competit praedicato tantum». Ibid.

27 Cfr. TS, 49-50 (90).

28 «Suppositio proprie dicta est proprietas termini subiecti ad praedicatum comparati». DPAL, p. 2.

29 «Suppositio est proprietas subiecti ad predicatum in propositione comparati». TS, p. 53 (93).

30 TS, p. 56 (95).

31 TS, p. 56(95). 
Un tercer punto de coincidencia en los tratados de Burleigh y Ferrer se observa en el tratamiento de los relativos dentro del tratado de la suposición. Con el paso del tiempo, la doctrina de la suposición gana en autonomía y absorbe cuestiones que con anterioridad se presentaban en capítulos distintos. Así ocurre con el tratado De relativis. En las Súmulas de Pedro Hispano ocupan un apartado especial, pero a partir del Tractatus De Suppositionibus de Burleigh es introducido dentro de las suposiciones. De esta manera, Guillermo de Ockham asume este tratamiento en su Summa Logicae, casi literalmente del tratado de su gran oponente escotista. ${ }^{32}$ Burleigh recoge en el De Puritate el mismo tratamiento presente en su primer tratado, que será también recogido de manera muy similar por S. Vicente Ferrer, en el capítulo VIII del Tractatus de Suppositionibus. ${ }^{33}$

Otra significativa coincidencia la encontramos en la inclusión en el Tractatus de Ferrer de la suposición colectiva, completamente novedosa en los tratados lógicos de su época, aunque en la tradición parisina se comienza a hablar del descenso copulativo a los singulares utilizando como ejemplo la proposición Omnes apostoli sunt duodecim, el mismo que Ferrer propone como ejemplo de suposición personal confusa y colectiva. ¿Qué relación tienen estos tratamientos ${ }^{34} \mathrm{De}$ nuevo, con cierta sorpresa constatamos una breve indicación en los tratados de Burleigh de este mismo ejemplo. ${ }^{35}$

De manera similar se podrían establecer paralelismos con Burleigh en el tratamiento de la suposición impropia, aunque Ferrer añade nuevas clases de este tipo de suposición. Otros puntos coincidentes han sido puestos de relieve por diversos autores. Asworth ha subrayado la similitud en el tratamiento de la suposición simple. ${ }^{36}$ Por su parte, Trentman sostiene que ambos autores tienen una semejante teoría de la predicación. ${ }^{37}$ Sin necesidad de ser exhaustivos, creo que las semejanzas son lo bastante significativas como para mostrar el influjo real de Burleigh en el tratado de las suposiciones de Ferrer.

Evidentemente no faltan las divergencias entre ambos planteamientos. Por ejemplo, Ferrer adopta un claro criterio clasificatorio, basado en el triple estado de la esencia, de tal manera que el tipo de suposición viene determinado por el tipo de predicación, y no por los supuestos. Esto quiere decir que el tipo de suposición viene determinada por el predicado. Por eso dice: «se hacen mal las divisiones de la suposición no tomándolas de parte del predicado, sino según los supuestos o significados, 0 de algo parecido». ${ }^{38} \mathrm{Y}$ alude de modo explícito a sus predecesores cuando dice: «no se hace bien o se describe la suposición personal cuando se dice: la suposición personal se da cuando el sujeto supone o se toma por sus supuestos, o aun cuando se diga por sus significados». ${ }^{39} \mathrm{Y}$

32 «William accepts and copies almost verbatim the whole of Walter's presentation of relative supposition». Brown, S. F., «Walter Burleigh's Treatise De Suppositionibus and its influence on William of Ockham», en Franciscan Studies, 32 (1972), p. 23.

33 TS pp. 163-172 (169-175). En este capítulo únicamente hay una sola cita al comienzo, en la que invoca la autoridad de Aristóteles. Por otro lado, las semejanzas con Burleigh y Ockham son patentes.

34 Cfr. Read, S. L., «Thomas of Cleves and Collective Supposition», pp. 73-75.

35 Walter Burleigh, DPAL, Tractatus brevoir (ed. Boehner), pp. 252-253.

36 Cfr. Ashworth, E.J., «The Doctrine of Supposition in the Sixteenth and Seventeenth Centuries», en Archiv für Geschichte der Philosophie, 51 (1969), p. 275.

37 Trentman, J.A., «Predication and Universals in Vincent Ferrer's Logic», en Franciscan Studies, 28 (1968), p. 52.

38 TS, p. 55 (94).

39 «Unde non bene sumitur seu describitur suppositio personalis quando dicitur: suppositio personalis est quando subiectum supponit seu accipitur pro sujs suppositis, seu etiam dicatur: pro suis significatis». TS, p. 55 (94). 
añade a continuación: «De la misma manera, no se define bien la suposición simple cuando se dice: hày suposición simple cuando el término o el sujeto se toma por su significado primero, o también cuando se dice, según la intención de la mente». ${ }^{40}$ Esas son, casi literales, las propuestas por Guillermo de Ockham ${ }^{41}$ y Walter Burleigh ${ }^{42}$ que Ferrer rechaza.

Para el dominico valenciano, toda suposición es significativa. Lo que sucede es que deben distinguirse distintos modos de significar. Un término puede tomarse con su significadọ material, como sucede en Homo est bisyllabum o con su significado formal, como en Homo est animal. Estos dos tipos de significación dan lugar a dos tipos de suposiciones, la material y la formal, respectivamente, pero sobre ellas puede hacerse una predicacion esencial (tendremos entonces una suposición natural) o accidental, ya sea por el ser en los singulares (suposición personal) o por el ser en el entendimiento (suposición simple).

Además, Ferrer aborda otra cuestión ampliamente debatida entre estos dos autores. Se trata de discernir la significación primera de un término. Para Ockham, la suposición de un término, como homo, es primeramente personal, pues lo primero representado por nuestro entendimiento es un individuo humano concreto. Sin embargo, para Burleigh, la suposición primaria es la simple, pues lo inmediatamente representado por el entendimiento es la naturaleza humana en abstracto. Nuestro autor resolverá la cuestión gracias a la distinción entre suposición común y discreta.

En efecto, para Ferrer el término común posee un modo de significar diverso al término discreto. En el primer caso, se presenta la naturaleza o esencia de la cosa de manera abstracta; en el segundo caso, el término discreto (Sócrates o «este hombre») significa la misma realidad, pero refiriéndose a ella de modo concreto y determinado. Así lo afirma explícitamente: «El término singular tiene otros modos de significar que el término común, [...]. Porque la naturaleza o esencia que el término común significa absolutamente y sin determinación, como el término homo; mientras que el término singular significa determinada y concretamente, como este término Sócrates». ${ }^{43}$

Sobre cada uno de estos términos puede realizarse una predicación esencial 0 accidental. Pero de modo primario, el término común designa la naturaleza humana, es decir, le conviene primeramente la suposición natural, mientras que al término discreto le corresponde con mayor propiedad la suposición personal. «Hay que saber que, aunque el término singular suponga naturalmente, simple y personalmente, como se ha dicho, sin embargo, principalmente le conviene más la suposición personal que la natural o la simple». ${ }^{44}$

40 «Similiter non bene sumitur suppositio simplex quando dicitur: suppositio simplex est quando terminus seu subiectum accipitur pro suo significato primo, sive etiam dicatur: pro intentione anime». TS, p. 55 (94).

41 «Suppositio personalis, universaliter, est illa quando terminus supponit pro suo significato». Guillermo de Ockham, Summa Logicae, I, c. 64, p. 195, lins. 4-5. «Suppositio simplex est; quando terminus supponit pro intentione animae, sed non tenetur significative». Summa Logicae, I, c. 64, p. 196. lins. 26-27 y 38-39.

42 «De primo dico, quod suppositio simplex est, quando terminus communis supponit pro suo significato primo vel pro omnibus contentis sub suo significato primo vel quando terminus singularis concretus vel terminus singularis compositus supponit pro suo significato totali, ut aliqualiter dictum est supra». Walter Burleigh, DPAL, p. 7.

43 «Propter quod notandum quod, ut dictum fuit superius, terminus semper supponit suum significatum. Terminus autem singularis alium modum significandi habet quam terminus communis, [...]. Nam naturam vel essentiam quam terminus communis significat absolute et non signate, ut iște terminus, homo, terminus singularis significat determinate et signate, ut iste terminus, Sortes». TS, p. 150 (160).

44 «Sciendum autem quod, quamvis terminus singularis supponat naturaliter, simpliciter et personaliter, ut est dictum, tamen principalius convenit sibi suppositio personalis quam naturalis vel simplex». TS, p. 151-152 (161-162). 
Obsérvese la riqueza que se contiene en el reconocimiento de diversos típos de significaciones (común y discreta, material y formal), frente al sentido unívoco de la significatio propuesta por Ockham. Según afirma Trentmam la doctrina significativa de Ockham puede ser definida como «monista», frente al «dualismo» de Burleigh y el «pluralismo» significativo de Ferrer. ${ }^{45}$ Toda esta teoría de la significación es novedosa, pero las referencias implícitas a Ockham y Burleigh parecen manifiestas. No es aventurado afirmar, por tanto, que Vicente Ferrer al componer su tratado tuviera delante las obras de estos dos autores, o al menos una recopilación de textos sobre la suposición elaborada por algún maestro en Artes de Oxford, como la de William Sutton.

\section{CONCLUSIÓN}

Llegados a este punto, es preciso sintetizar los datos que hemos señalado en nuestra exposición. Sin pretender ser exhaustivos en el análisis comparativo de los textos, estamos en condiciones de plantear con cierto fundamento una hipótesis que deberá ser corroborada ulteriormente, acerca de las fuentes del tratado de Ferrer.

El Tractatus De Suppositionibus de Vicente Ferrer debe entenderse como el resultado de la sistematización de la ontología de Santo Tomás, pero en términos de teoría de la suposición. En la tradición tomista, una vez reconocido el valor indiscutible de su autoridad doctrinal, quiere encontrarse un tratamiento completo de las doctrinas lógicas de su tiempo. Es interesante constatar cómo proliferaron las obras lógicas atribuidas a Santo Tomás, comprobándose posteriormente como inauténticas (por ejemplo la Summa totius Logicae) o de autenticidad dudosa (De Fallaciis). ${ }^{46} \mathrm{En} \mathrm{mi}$ opinión durante el siglo XIV, en la tradición dominicana se vio la necesidad de colmar la laguna de obras lógicas de Santo Tomás, porque de algún modo se llegó a la convicción de que detrás del instrumento lógico se encontraban presentes las grandes cuestiones metafísicas. Un buen ejemplo de ello es la teoría de la suposición de los términos que contaba con sistematizaciones nominalistas y escotistas, pero no tomistas.

Vicente Ferrer acometió la tarea de proponer la versión tomista sobre la teoría de la suposición. Para ello se inspiró en los tratados lógicos de Aristóteles y Tomás de Aquino, pero se sirvió además de las sistematizaciones de los lógicos inmediatamente anteriores, como la llevada a cabo por William Sutton. ${ }^{47}$ Quizás accediera directamente a los tratados de Burleigh y Ockham, o bien trabajara con textos o tratados de autores procedentes de la Facultad de Artes de Oxford, que se inspiraron en las polémicas entre el Venerabilis Inceptor y el Doctor Planus. ¿Utilizó Ferrer los materiales de Juan Linconiense (su nombre induce a pensar en su ascendencia inglesa), el prácticamen-

45 «The complexity of Vincent's ideas is striking enough, however, that one might say without too much oversimplification that Burleigh was a kind of semantic dualist and Ockham a monist, Ferrer was pluralist». Trentman, J. A., «The Idea of Signification in Vincent Ferrer's Logic», p 1302.

46 Las últimas investigaciones parecen inclinarse hacia la inautenticidad de esta obra atribuida a Tomás de Aquino, cfr. Gauthier, R-A., «Introducción», Sanctae Thomae. Opera Omnia (I), Roma, Editio Leonina \& J. Vrin, 1989, p. 57.

47 Un hecho muy significativo resulta la coincidencia de que uno de los tres manuscritos que se conservan del Tractatus de Suppositionibus de Ferrer, se encuentra en el convento dominicano de Viena, en donde se halla también el tratado De Suppositionibus de Sutton y una cuestión De Universalibus atribuida a Walter Burleigh. 
te desconocido maestro de Ferrer en Lérida y autor de algunos tratados de lógica que no han llegado hasta nosotros? Toda conjetura en este sentido carece hoy por hoy del necesario respaldo documental. No obstante, los textos de William Sutton en los que se recoge la polémica entre Ockham y Burleigh invitan a pensar en la incidencia de la lógica de la Facultad de Artes de Oxford en el incipiente pensamiento filosófico de la Corona de Aragón.

Pero la historia de la filosofía no puede ser reducida a análisis textuales de las fuentes utilizadas. No toda la filosofía de Tomás de Aquino estaba ya escrita en Aristóteles; ni Escoto estaba todo contenido en Buenaventura: ni Vicente Ferrer se encuentra en el Doctor Angélico, ni en Burleigh, ni en Ockham. Hacía falta la creatividad personal, la asimilación intelectual de la propia tradición, abierta a las nuevas aportaciones de las distintas corrientes filosóficas de su momento. Esta es, en último término, la clave interpretativa para comprender las originales propuestas semánticas de Ferrer.

José Angel García Cuadrado

Facultad Eclesiástica de Filosofía

Universidad de Navarra

31009 Pamplona 